\title{
Early Attentional Deficits in an Attention-to-Prepulse Paradigm in ADHD Adults
}

\author{
Annette Conzelmann, Paul Pauli, Ronald F. Mucha, Christian P. Jacob, Antje B. M. Gerdes, \\ Jasmin Romanos, Christina G. Bähne, Monika Heine, Andrea Boreatti-Hümmer, Georg W. Alpers, \\ Andreas J. Fallgatter, Andreas Warnke, Klaus-Peter Lesch, and Peter Weyers \\ University of Würzburg
}

\begin{abstract}
Adults with attention-deficit/hyperactivity disorder (ADHD) were examined for early and late attentional processes as a function of controlled attention. The test paradigm was the attentional modulation of prepulse inhibition (PPI; early controlled attentional processing) and prepulse facilitation (PPF; late controlled attentional processing). In 49 patients and 49 controls, the authors measured acoustic startle responses to 96-dB startle pulses preceded 120, 240 (for PPI), 2,000, and 4,500 (for PPF) ms by a 68-dB prepulse noise. Geometric figures signaled that prepulses were to be ignored or attended to (automatic vs. controlled attention). ADHD patients exhibited deficits in prepulse modulation, but these reflected an interaction of controlled attention and time of information processing. Normal PPI and PPF occurred under all conditions except for controlled attentional modulation of PPI. Attention deficits in ADHD patients may reflect not general derangements in information processing or ability to attend but, rather, selective disturbances of controlled attention during early information processing.
\end{abstract}

Keywords: startle response, ADHD, attention, prepulse facilitation, prepulse inhibition

\section{ADHD: A Disorder of Deficient Subcomponents of Attentional Processing}

Attention-deficit/hyperactivity disorder (ADHD) is a prevalent form of abnormal behavior in children; $30-60 \%$ of affected individuals also show ADHD symptoms in adulthood (Wender, Wolf, \& Wasserstein, 2001). Attentional dysfunction is one of the core symptoms (Douglas, 1999). For at least two reasons, to understand it, one may need to acknowledge that attentional functioning embodies multiple components.

Annette Conzelmann, Paul Pauli, Ronald F. Mucha, Antje B. M. Gerdes, Georg W. Alpers, and Peter Weyers, Department of Psychology (Biological Psychology, Clinical Psychology, and Psychotherapy), University of Würzburg, Würzburg, Germany; Christian P. Jacob, Christina G. Bähne, Monika Heine, Andrea Boreatti-Hümmer, Andreas J. Fallgatter, and KlausPeter Lesch, Department of Psychiatry and Psychotherapy, University of Würzburg; Jasmin Romanos and Andreas Warnke, Department of Child and Adolescent Psychiatry and Psychotherapy, University of Würzburg.

Andreas J. Fallgatter is now at the Department of Psychiatry, University of Tübingen, Tübingen, Germany. This work was supported by German Research Foundation Grant DFG (KFO 125/1) awarded to Paul Pauli, Ronald F. Mucha, Peter Weyers, Christian Jacob, Armin Schmidtke, Andreas Fallgatter, and Klaus-Peter Lesch and European Commission Grant NEWMOOD LSHM-CT-2003-503474 awarded to Klaus-Peter Lesch. We thank R. Gerhard for assisting with data acquisition and preprocessing. All authors report no biomedical financial interest or potential conflicts of interest.

Correspondence concerning this article should be addressed to Peter Weyers, Department of Psychology (Biological Psychology, Clinical Psychology and Psychotherapy), University of Würzburg, Marcusstr. 9-11, 97070 Würzburg, Germany. E-mail: weyers@psychologie.uniwuerzburg.de
First, recent theories on ADHD (Sergeant, Geurts, Huijbregts, Scheres, \& Oosterlaan, 2003) suggest that the attentional dysfunctions in ADHD may reflect executively determined functions. Thus, ADHD may be affected by deficient top-down control rather than bottom-up automatic processing. Indeed, ADHD patients show reduced volumes of the prefrontal and the anterior cingulate cortex (Seidman et al., 2006), areas responsible for top-down attentional control (MacDonald, Cohen, Stenger, \& Carter, 2000). In addition, ADHD boys showed event-related potentials that were reduced only in the case of attended but not ignored stimuli (Satterfield, Schell, \& Nicholas, 1994). Because paying attention to the stimuli was actually controlled by the patients themselves, this suggests an important role for controlled attention and that the deficit may not arise for responses that occur with less control (more automatic).

Second, disturbed attentional processes in ADHD that are under top-down control appear to be sensitive to temporal parameters. Thus, ADHD children showed a dysfunction in visual selective attention in early but not later event-related potentials (Jonkman, Kenemans, Kemner, Verbaten, \& van Engeland, 2004). ADHD patients appear to have problems with time-based attentional resolution when the stimuli are presented at very short time intervals (Bellgrove et al., 2006). This suggests that early top-down controlled attentional processes may be slowed down but that later top-down controlled attentional processes may be intact. This might also explain the slower performance of ADHD patients (Nigg, Blaskey, HuangPollock, \& Rappley, 2002).

Taking these two points together, ADHD may reflect a dysfunction in early but not late top-down controlled attentional processes. At the same time, automatic bottom-up early and late information processing may be normal. 


\section{The Prepulse Startle Paradigm to Dissect Subcomponents of Attentional Processing}

A paradigm ideally suited for dissecting attentional processing is the prepulse modulation of the startle response. The startle response is a reflexive response evoked by a sudden noise. In humans, it is recorded electromyographically from the orbicularis oculi muscle (Blumenthal et al., 2005). On a test trial, a weak nonstartling acoustic stimulus, the prepulse, precedes the startle stimulus at different time intervals (stimulus onset asynchronies, SOAs). Modulation is then simply derived by contrasting startle responses preceded with the prepulse to those preceded without the prepulse (Graham, 1975).

Through judicious choice of two variables (the SOAs and the degree of control of attending to the prepulse), it is possible with the prepulse-modulation paradigm to systematically model at least four stages of attentional processing (see Figure 1). These can be argued to index bottom-up automatic and top-down controlled processing during early and at late periods of information processing (cf. Jennings, Schell, Filion, \& Dawson, 1996). Thus, two stages reflect a noncontrolled processing of the information in the prepulse (Schell, Wynn, Dawson, Sinaii, \& Niebala, 2000). In such test conditions, a participant is typically not instructed to pay special attention to the prepulse. An early preattentional stage of processing is seen as prepulse inhibition (PPI; see Figure 1A). This is the inhibited startle response observed with short SOAs between $30 \mathrm{~ms}$ and $500 \mathrm{~ms}$ (Blumenthal, 1999). This inhibition is believed to protect information processing because it ensures that the processing of the prepulse stimulus is not disrupted by the startle stimulus. This effect thereby indexes early automatic bottom-up sensory gating and is even observable during sleep (Silverstein, Graham, \& Calloway, 1980). PPI deficits leading to sensory overload and deficient filtering of irrelevant information have been found in several psychiatric disorders with attentional dysfunctions, such as Tourette's syndrome, enuresis, schizophrenia, obsessive-compulsive disorder, and Huntington's disease (Braff, Geyer, \& Swerdlow, 2001)
A second stage of automatic bottom-up information processing is reached at a later time period (see Figure 1B). This is the facilitated startle response with SOAs beginning about $800 \mathrm{~ms}$ after the prepulse presentation and is termed prepulse facilitation (PPF). This phenomenon probably reflects automatic orienting and activation of the sensory modality of the prepulse (Graham, 1975; Putnam \& Vanman, 1999). Deficits in PPF suggest reduced allocation of processing resources to stimuli and were found in schizophrenia patients (Wynn et al., 2004).

Third and fourth stages of prepulse modulation can be achieved when PPI and PPF are tested in conditions of controlled attention. This is often achieved by instructing participants to actively attend to the prepulse information and can be seen as top-down controlled attentional processing. Testing under controlled attention serves to magnify the effects of the acoustic prepulse: In the short run, it magnifies suppression (PPI; see Figure 1C), and in the long run, it magnifies facilitation (PPF; see Figure 1D; Blumenthal, 1999; Filion, Dawson, \& Schell, 1993; Putnam \& Vanman, 1999). From a functional perspective, the attentional increase in PPI likely reinforces the protected processing of the prepulse in the sense of early selective attention. The attentional increase in PPF can be seen to increase orienting to the modality of the prepulse and therefore reflects the sustained version of selective attention. Accordingly, these effects would refer to top-down early and late controlled attentional processing, respectively. At present, only patients with schizophrenia were found to be marked by a lack of both an attention effect on PPI and on PPF (Hazlett et al., 2007).

Accordingly, different forms of prepulse modulation offer a simple and comparative paradigm to index early and late bottom-up automatic and top-down controlled attentional processing (Jennings et al., 1996). The effects are robust, and the effect size is strong. The model can be applied to developmental, neuropsychological, and animal work (Blumenthal, 1999; Putnam \& Vanman, 1999). Furthermore, in contrast to classical measures of dysfunctional attention in ADHD, such as performance in the continuous performance test (Song, Shin, Jon, \& Ha, 2005) or a

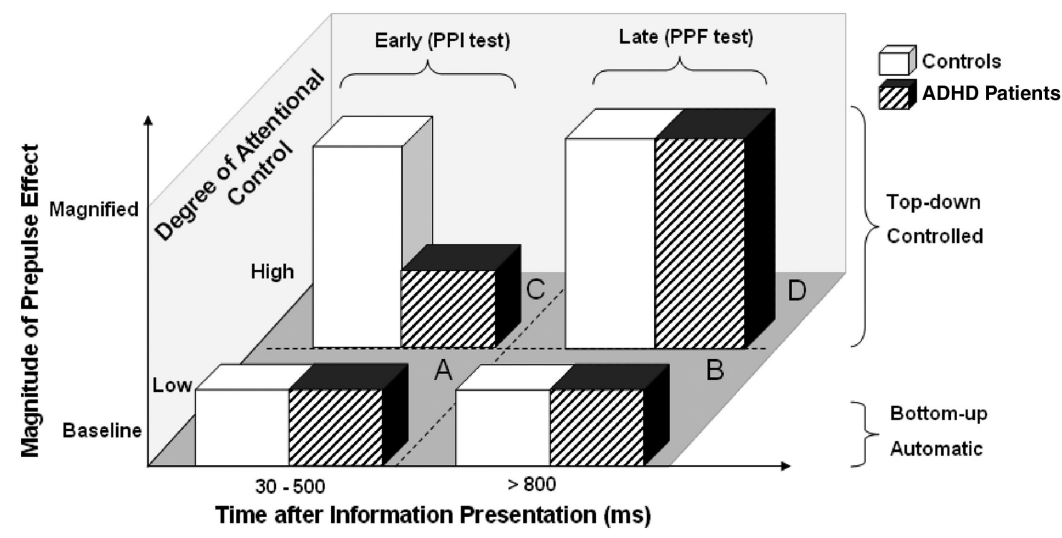

Figure 1. A hypothetical model of prepulse modulation ( $y$ axis) for early and late periods ( $x$ axis) of information processing under conditions of ignoring and paying attention to the prepulse ( $z$ axis) in controls and attention-deficit/hyperactivity disorder (ADHD) patients. A. Early bottom-up automatic preattentional processing, automatic prepulse inhibition (PPI), sensory gating. B. Late bottom-up automatic attentional processing, automatic prepulse facilitation (PPF), orienting. C. Early top-down controlled attentional processing, controlled PPI, early selective attention. D. Late top-down controlled attentional processing, controlled PPF, sustained selective attention. 
delayed attentional blink (Li, Lin, Chang, \& Hung, 2004), prepulse paradigms allow the isolation of specific attentional mechanisms, as indexed in Figure 1.

\section{Prepulse Startle Paradigms in ADHD}

Existing studies looking at prepulse phenomena in ADHD patients typically focused on bottom-up automatic PPI. This bottom-up automatic PPI in ADHD reflecting normal sensory gating functions appears to be intact. Three studies compared healthy controls with ADHD boys comorbid with Tourette's syndrome (Castellanos et al., 1996) or with enuresis (Ornitz, Hanna, \& de Traversay, 1992). However, PPI deficits were attributed to the comorbid disorders and not to ADHD. Two other studies examined adult ADHD patients without comorbidities (Feifel, Minassiana, \& Perrya, 2009; Hanlon, Karayanidis, \& Schall, 2009), but again no PPI deficits could be found.

There is only one study addressing top-down controlled attentional PPI and PPF. Hawk, Yartz, Pelham, and Lock (2003) revealed in boys with ADHD dysfunctional top-down controlled attention modulated PPI but normal bottom-up automatic PPI and PPF. However, the results with PPF were not fully interpretable, as the healthy participants showed no attentional modulation of PPF. A wider range of experimental protocols can be used in adults with ADHD, because their compliance is more predictable than for children. Considering that attentional symptoms are more stable than hyperactive-impulsive symptoms in ADHD in adulthood (Biederman, Mick, \& Faraone, 2000), their in-depth examination is indicated.

\section{Aim of the Study}

Taken together, previous prepulse studies suggest a specific deficit in ADHD patients, marked by a dysfunction in early topdown controlled attentional processes (see Figure 1C). In line with this, attentional PPI modulation was found to be associated with thalamic and prefrontal activity (Hazlett et al., 1998, 2001). And, as outlined above, ADHD patients show abnormalities in these structures. In addition, the assumption about disturbed early attentional control is supported by EEG studies revealing deficits in controlled but not automatic conditions and in early but not late event-related potentials, as described above.

Therefore, the present study was designed to address the hypothetical pattern of dysfunctions at early but not late controlled attentional processing in ADHD depicted in Figure 1. We addressed this on the basis of a within-subject, two variable, prepulse modulation paradigm applied to two groups of participants, comprising adults of both genders with and without ADHD. Statistically, we expected the absence of an attentional effect on PPI in ADHD patients, whereas ADHD patients and healthy controls should show comparable attentional modulation of PPF.

\section{Method}

\section{Participants}

Recruitment and assessment. This study was part of a large ADHD project established at the University of Würzburg, Würzburg, Germany. Employed were adult outpatients from the Depart- ment of Psychiatry and Psychotherapy, referred for diagnostic ADHD assessment and treatment. Many of them came to the department because ADHD was diagnosed in their children and they recognized the symptoms in themselves; others were recommended by their family doctors. Altogether, 1,021 persons were evaluated. Five hundred twelve control participants were recruited via advertisement. These were first evaluated during a telephone call to ensure the absence of psychiatric symptoms. They were given a short interview to assess Diagnostic and Statistical Manual of Mental Disorders (4th ed.; DSM-IV; American Psychiatric Association, 1994) psychiatric disorders. One hundred eighty-six could be recruited for further diagnostic assessment. Three hundred twenty-six persons had to be ruled out (107 were suspicious for psychiatric disorders, 80 refused to participate in further studies, and 139 did not allow matching to the clinical sample according to sociodemographic criteria).

The current diagnoses relevant for this study were established by two experienced psychiatrists who conducted the structured interview independently of each other on two appointments carried out on the same calendar day (Jacob et al., 2007). The clinicians were required to agree on their diagnostic evaluation and the decision about the inclusion of the participants in the final sample. If the estimates of the two investigators did not match, the issue was discussed and reevaluated with additional questions until consensus was reached. The screening was carried out with a structured clinical interview based on the DSM-IV symptom list for ADHD; this provided information on current ADHD symptoms and on ADHD symptoms present during the participants' childhood. In addition, participants were asked to describe these symptoms in detail to ensure that these symptoms were reliable. They had to be obvious in at least two situations and to be accompanied by impairment; it was also determined whether these symptoms were not due to other psychiatric disorders (Criteria B to E of the $D S M-I V$ criteria for ADHD). School reports were also evaluated. Additional information from relatives was considered whenever available, but this was not obligatory. Unresolved contradictory information led to exclusion of the potential participants. In addition, we obtained a self-report of ADHD symptoms during childhood with the Wender Utah Rating Scale (WURS-k; RetzJunginger et al., 2002). Based on a sample of patients of various psychiatric disorders, split half reliability of the WURS-k was $0.85, \alpha$ was 0.91 , and a cut off of 30 led to a specificity of $76 \%$ and sensitivity of $85 \%$, to differentiate this sample with respect to the presence of childhood ADHD. These data were obtained to get additional diagnostic information, but because ADHD patients tend to underreport their symptoms (Murphy \& Barkley, 1996), the clinicians' evaluations of ADHD symptoms were the final criterion for ADHD diagnosis. However, when the analyses for PPI and PPF were carried out with patients above the WURS-k cutoff of 30 $(n=26)$ and controls below the cut off of $30(n=46)$, all effects remained as reported in the Results section. Current ADHD symptoms of our study sample were rated by a self-report of the severity of the DSM-IV symptoms of ADHD on a scale from 0 to 3 . This assessment indicated that ADHD patients showed a strong impairment (see Table 1). Normative ratings for adult symptoms were not assessed.

We also assessed comorbid Axis I and II disorders using the Structured Clinical Interview for DSM-IV Disorders (SCID I and SCID II; First, Spitzer, Gibbon, \& Williams, 1996, 1997). Partic- 
Table 1

Sociodemographic and Psychometric Data of Healthy Controls and ADHD Patients

\begin{tabular}{|c|c|c|c|c|c|c|c|}
\hline \multirow[b]{2}{*}{ Variable } & \multicolumn{3}{|c|}{ Controls $(n=49)$} & \multicolumn{3}{|c|}{$\operatorname{ADHD}(n=49)$} & \multirow[b]{2}{*}{ Statistics } \\
\hline & $M$ & $S D$ & $\%$ & $M$ & $S D$ & $\%$ & \\
\hline Age (years) & 35.2 & 9.6 & & 35.3 & 10.1 & & $t(96)=0.04, p=.968$ \\
\hline IQ (MWT-B) & 119.3 & 13.9 & & 114.3 & 14.6 & & $t(96)=1.7, p=.084$ \\
\hline WURS & 12.2 & 9.5 & & 31.0 & 15.1 & & $t(96)=7.4, p<.001$ \\
\hline Self-reported ADHD severity & 9.5 & 5.3 & & 26.4 & 9.6 & & $t(96)=10.8, p<.001$ \\
\hline $\mathrm{C}$ Inattentiveness $(D S M-I V)$ & 0.8 & 1.2 & & 6.6 & 1.8 & & $t(96)=18.0, p<.001$ \\
\hline C Hyperactivity/Imp. (DSM-IV) & 0.8 & 1.2 & & 5.0 & 2.7 & & $t(96)=10.1, p<.001$ \\
\hline A Inattentiveness $(D S M-I V)$ & 1.1 & 1.6 & & 7.2 & 1.1 & & $t(96)=22.0, p<.001$ \\
\hline A Hyperactivity/Imp. $(D S M-I V)$ & 1.4 & 1.2 & & 5.4 & 2.4 & & $t(96)=10.4, p<.001$ \\
\hline Bipolar NOS & & & 0 & & & 2.0 & $\chi^{2}(1, N=98)=1.0, p=.315$ \\
\hline Major depression & & & 0 & & & 6.1 & $\chi^{2}(1, N=98)=3.1, p=.079$ \\
\hline Dysthymic disorder & & & 0 & & & 2.0 & $\chi^{2}(1, N=98)=1.0, p=.315$ \\
\hline GMC & & & 0 & & & 2.0 & $\hat{\chi}^{2}(1, N=98)=1.0, p=.315$ \\
\hline Depressive NOS & & & 0 & & & 10.9 & $\chi^{2}(1, N=98)=5.2, p=.022$ \\
\hline Panic disorder & & & 0 & & & 2.0 & $\chi^{2}(1, N=98)=1.0, p=.315$ \\
\hline Agoraphobia & & & 0 & & & 2.0 & $\chi^{2}(1, N=98)=1.0, p=.315$ \\
\hline Social phobia & & & 0 & & & 4.1 & $\chi^{2}(1, N=98)=2.0, p=.153$ \\
\hline Specific phobia & & & 0 & & & 4.1 & $\chi^{2}(1, N=98)=2.0, p=.153$ \\
\hline PTSD & & & 0 & & & 4.1 & $\chi^{2}(1, N=98)=2.0, p=.153$ \\
\hline Addiction & & & 0 & & & 12.2 & $\chi^{2}(1, N=98)=6.4, p=.011$ \\
\hline Smoking & & & 14.3 & & & 32.7 & $\hat{\chi}^{2}(1, N=98)=4.6, p=.032$ \\
\hline Eating disorder & & & 0 & & & 2.0 & $\chi^{2}(1, N=98)=1.0, p=.315$ \\
\hline Paranoid PD & & & 0 & & & 8.2 & $\chi^{2}(1, N=98)=4.2, p=.041$ \\
\hline Antisocial PD & & & 0 & & & 8.2 & $\chi^{2}(1, N=98)=4.2, p=.041$ \\
\hline Borderline PD & & & 0 & & & 10.2 & $\chi^{2}(1, N=98)=5.3, p=.022$ \\
\hline Histrionic PD & & & 0 & & & 28.6 & $\chi^{2}(1, N=98)=16.3, p<.001$ \\
\hline Narcissistic PD & & & 0 & & & 20.4 & $\chi^{2}(1, N=98)=11.1, p=.001$ \\
\hline Avoidant PD & & & 0 & & & 18.4 & $\chi^{2}(1, N=98)=9.9, p=.002$ \\
\hline Negativistic PD & & & 0 & & & 4.1 & $\chi^{2}(1, N=98)=2.0, p=.153$ \\
\hline Depressive PD & & & 0 & & & 4.1 & $\chi^{2}(1, N=98)=2.0, p=.153$ \\
\hline Obsessive-compulsive PD & & & 0 & & & 16.3 & $\chi^{2}(1, N=98)=4.0, p=.045$ \\
\hline Any Axis I comorbidity & & & 0 & & & 40.8 & $\chi^{2}(1, N=98)=25.1, p<.001$ \\
\hline Any Axis II comorbidity & & & 0 & & & 67.3 & $\chi^{2}(1, N=98)=49.8, p<.001$ \\
\hline Overall comorbidity & & & 0 & & & 77.6 & $\chi^{2}(1, N=98)=62.1, p<.001$ \\
\hline
\end{tabular}

Note. $\quad$ ADHD $=$ attention-deficit/hyperactivity disorder; MWT-B = Multiple Choice Word Fluency Test (IQ test; Lehrl, 1989); WURS = Wender Utah Rating Scale (Retz-Junginger et al., 2002); Self-reported ADHD severity = current self-reported severity of Diagnostic and Statistical Manual of Mental Disorders (4th ed.; DSM-IV; American Psychiatric Association, 1994) ADHD symptoms on a 0 to 3 scale; C Inattentiveness $(D S M-I V)=$ number of childhood ADHD symptoms of inattentiveness according to DSM-IV, expert rating; C Hyperactivity/Imp. (DSM-IV) = number of childhood ADHD symptoms of hyperactivity/impulsivity according to $D S M-I V$, expert rating; A Inattentiveness $(D S M-I V)=$ number of current ADHD symptoms of inattentiveness according to $D S M-I V$, expert rating; A Hyperactivity/Imp. $(D S M-I V)=$ number of current ADHD symptoms of hyperactivity/impulsivity according to $D S M-I V$, expert rating; NOS = not otherwise specified; GMC = mood disorder due to a general medical condition; PD = personality disorder.

ipants also completed the Multiple Choice Word Fluency Test (MWT-B; Lehrl, 1989) as a valid estimator of crystalline intelligence. The MWT-B is a widely used German test requesting the individual to select for each of the 37 items the only meaningful term among five given options. This test shows construct validity with the HAWIE vocabulary test of .95 and a test-retest reliability of .90 .

Exclusion criteria for both groups were the following: age below 18 or above 60 years, IQ below 80 (exclusion of five potential ADHD patients), severe somatic disorders (exclusion of 21 potential ADHD patients), or not European nationality (exclusion of 19 potential ADHD patients and five potential controls). Further exclusion criteria for patients were unvalidated current adult (exclusion of 39 potential ADHD patients) and childhood ADHD (exclusion of seven potential ADHD patients) according to the $D S M-I V$ symptom list with an onset before the age of 7 years, severe impairment and evidence of being impaired in at least two settings, or symptoms better accounted for by another mental disorder (exclusion of 110 potential ADHD patients). Not included in the control group were individuals with a lifetime or any current SCID I or SCID II diagnosis (exclusion of 19 potential controls) or more than three fulfilled DSM-IV ADHD criteria of current or lifetime symptoms of inattentiveness or hyperactivity/impulsivity (exclusion of 24 potential controls).

Finally, the ADHD project consisted of 820 ADHD patients and 138 controls. The present participants were recruited from this pool, resulting in 75 adult ADHD patients and 82 controls. The reported data are based on a final sample of $49 \mathrm{ADHD}$ patients and 49 controls, as many original recruits were excluded for various reasons: current antidepressant medication (12 patients), less than two valid startle data points per experimental condition (seven patients and one control), startle nonresponders having more than $33 \%$ trials with zero reactions (Sobin, Kiley-Brabeck, \& Karayiorgou, 2005; five patients and seven controls), loss of the matching 
partner (we individually matched for gender and age given suggestions that gender and age influence prepulse startle modulation; Ellwanger, Geyer, \& Braff, 2003; Swerdlow et al., 1993; two patients and 25 controls). Carrying out the analyses of PPI and PPF after additionally including these latter 27 participants revealed the same pattern of results as reported below.

Written informed consent was obtained from all participants. Participants were paid 40 Euros for taking part in the diagnostic and experimental assessment.

Final sample. Table 1 shows sociodemographic and psychometric data for ADHD patients and controls. In each group, there were 24 women. Of the 49 ADHD patients, 31 patients were of the ADHD combined type and 18 of the ADHD inattentive type. The ADHD patients also showed a number of comorbid psychiatric disorders, comparable to previously published data from this population (Kessler et al., 2006). Our two experimental groups were comparable with respect to age, IQ, and sex. The self-report ADHD symptoms during childhood, the self-report adult ADHD severity score and the clinicians' ratings of current and childhood ADHD symptoms of inattentiveness and hyperactivity were higher in patients compared with controls.

All participants were free from any ADHD-specific medication for at least four days. The 13 patients who were on methylphenidate agreed to discontinue the medication after the risks had been discussed with their psychiatrists.

\section{Experimental Session}

The participants were seated $0.7 \mathrm{~m}$ in front of the monitor presenting the experimental instructions. After assessing sociodemographic and psychometric characteristics, the electrodes were applied. Acoustic stimuli were presented against a background of continuous $60-\mathrm{dB}$ white background noise (created by a white noise generator; Lafayette Instruments, Lafayette, IN). The experimental procedures were computer controlled (ERTS, Version 3.32, BeriSoft, Frankfurt, Germany). Startle and prepulse stimuli were elicited by a Contact Precision Instruments noise generator (London, England) and presented binaurally over headphones (Beyerdynamic DT 331, Heilbronn, Germany).

The participants were first presented with three startle stimuli to verify the quality of recording and to get the participants accustomed to the probe. Startle stimuli consisted of bursts of $50 \mathrm{~ms}$ of 96-dB white noise with an instantaneous rise time.
Following this, the participants were instructed on the timediscrimination task. They had to discriminate the length of a 5-s or 7-s time interval, which was delimited by the prepulse and a second presentation of the same tone at the end of the interval (20-ms, $68-\mathrm{dB}$ white noise burst with $20-\mathrm{ms}$ rise time). A button was to be pressed when the interval was long and not pressed when it was short. Following this, the controlled attention condition was explained. This was signaled by one of two geometric figures presented at the beginning of each trial (white silhouetted square or triangle on black background, size $194 \times 191$ pixels). One indicated that the time-discrimination task was required, and therefore, attention was allocated to the prepulse (attention condition). After the other figure, the time-discrimination task was not required, and stimuli were ignored during this trial (ignore condition).

The participants then performed eight training trials (each of the four combinations of interval duration and attention condition were presented twice). These trials were comparable to the trials of the main part of the study (see Figure 2), except that no startle stimulus was presented, and feedback was provided in the intertrial intervals using the words "correct" (button press in the attend condition at the 7-s interval) and "false" (button press in the ignore condition or attend condition at the 5-s interval).

The main part of the study investigated short SOAs (120 and $240 \mathrm{~ms}$ ) to elicit PPI (early attentional processing) and long SOAs (2,000 and 4,500 ms) to elicit PPF (late attentional processing), as reported previously (Filion et al., 1993; Hawk et al., 2003; Hazlett et al., 2007). The test session had 48 trials (see Figure 2). Twentyfour trials had to be attended, 24 had to be ignored. Of these respective test trials, 16 consisted of four trials for each SOA condition with two trials having a time interval of $5 \mathrm{~s}$ and two of $7 \mathrm{~s}$. The remaining eight were considered filler trials, entailing four trials without a startle stimulus (two trials each with the 5-s and the 7-s time interval and four trials presenting the geometric figure and a startle stimulus without a prepulse). Twelve startle stimuli were also presented alone during the intertrial intervals (without any geometric figure or prepulse). These were used to define basic startle reactions and to calculate startle prepulse modulation.

Six pseudorandom sequences of trials were established; half of them used the triangle to indicate the attention condition, the other half used the square. The sequences had the following constraints: no more than three trials of the same attention condition or more than three consecutive alterations of conditions followed each other (e.g., $\square \Delta \square \Delta \square \Delta$ or $\Delta \Delta \square \square \Delta \Delta$ ). In addition, there were no

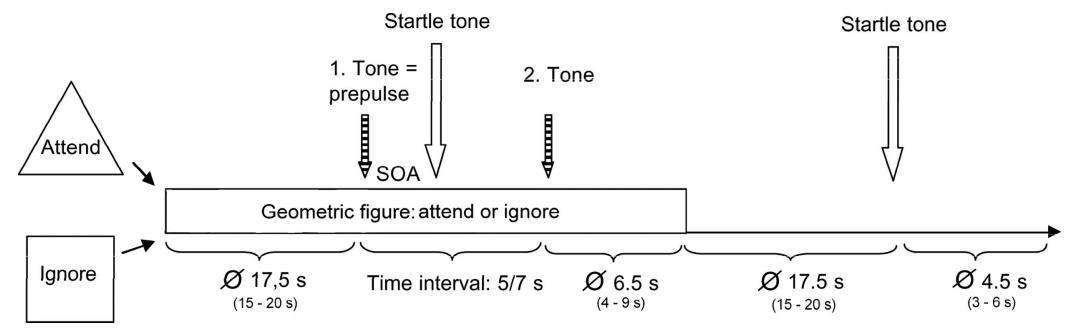

Figure 2. Example of one trial with time estimation interval, the latter beginning with the prepulse stimulus and followed by the startle tone. After each trial, a black screen was presented ending with the beginning of the next trial (intertrial interval). During intertrial intervals, a startle tone was occasionally presented. SOA $=$ stimulus onset asynchrony. 
consecutive intervals of the same length or the same SOA (Hawk et al., 2003). Finally, the test session was divided into two equivalent blocks separated by a short break; the experimental conditions were evenly distributed over the two blocks.

\section{Startle Measurement}

Startle responses were sampled electromyographically from the orbicularis oculi and were recorded digitally with 1,000 Hz (Psylab, Contact Precision Instruments bio amplifier, London, England, and Hellige miniature electrodes filled with electrode gel). Signals were filtered with high- and low-pass filters at $30 \mathrm{~Hz}$ and $500 \mathrm{~Hz}$, respectively, and a notch filter of $50 \mathrm{~Hz}$.

The EMG signals were analyzed offline with an interactive computer program (Schulz \& Alpers, 2007) written in Matlab (MathWorks, München, Germany). The data were first rectified and integrated and then smoothed using a time constant of $100 \mathrm{~ms}$. Amplitude was quantified as the difference between the baseline (average over $20 \mathrm{~ms}$ before the startle probe) and the peak occurring within 21 to $150 \mathrm{~ms}$ after startle probe onset. Trials were excluded when a spontaneous eye-blink response was detected during the baseline or within $20 \mathrm{~ms}$ after the startle probe presentation. A trial was also excluded in case the baseline or the startle response deviated more than three standard deviations from the individual's mean $(6.89 \%$ of trials). Trials with no detectable response $(8.07 \%$ of trials) were included in analyses with amplitude of zero.

For each SOA by attention combination, EMG magnitude averages were used to calculate percent prepulse inhibition (PPI) and facilitation (PPF) relative to intertrial interval (ITI) startle magnitude according to the common procedure ([$\left.M_{\text {prepulse }}-M_{\mathrm{ITI}}\right) /$ $\left.\left(M_{\text {ITI }}\right)\right] \times 100$; Hawk et al., 2003).

\section{Statistical Analysis}

Data were analyzed with SPSS 15 . Separate repeatedmeasurement analyses of variance (ANOVAs) were conducted for PPI and PPF with the between-subjects factor group (ADHD, controls) and the within-subject factors SOA (120, 240, or 2,000, 4,500 ) and attention (attend, ignore). Baseline startle responses and their habituation were evaluated with repeated-measurement ANOVAs with the between-subjects factor group and the withinsubject factor time of measurement (T1, T2, T3, T4; the 12 ITI startle responses were divided into four measurement time points, each being the mean of three consecutive startle responses).

Questionnaire data and overall error rate for the timediscrimination task were evaluated with one-way ANOVAs with the between-subjects factor group. We compared false alarms (button press in the ignore condition or the 5-s attend condition) and misses (no button press in the 7-s attend condition) with an ANOVA using error type (false alarms/misses) as within-subject and group as between-subjects factors. Errors in ignore (button press) and attend trials (no button press for the 7-s interval, button press in the 5-s interval) were also compared for PPI and PPF with an ANOVA using prepulse condition (PPI/PPF) and attention (ignore/attend) as within-subject factors and group as betweensubjects factor.

Finally, we also conducted all analyses with gender as betweensubjects factor to control for possible gender influences. Because gender never had a significant impact, the results were reported without the factor gender. Greenhouse-Geisser corrections were applied where necessary. Follow-up $t$ tests were Bonferroni corrected, and we report adjusted $p$ values.

\section{Results}

\section{Prepulse Inhibition (Early Attentional Processing)}

Table 2 depicts the statistical effects for the prepulse inhibition ANOVA. PPI scores are depicted in Table 3. The ANOVA did not indicate a significant group difference in PPI between ADHD patients and controls, nor a significant attention effect. As main finding, however, ANOVA revealed a significant Group $\times$ Attention interaction, which indicated deficient controlled attentional processing in ADHD patients. Only controls showed the normal attention effect with stronger PPI in the attend compared with the ignore condition; this was not present in ADHD patients. Furthermore, the SOA effect was significant, which was due to a stronger prepulse inhibition for SOA 120 compared with SOA 240.

\section{Prepulse Facilitation (Late Attentional Processing)}

Table 2 also depicts the statistical effects for the prepulse facilitation ANOVA, and Table 3 shows the corresponding PPF means. The ANOVA for PPF failed to indicate any significant Group $\times$ Attention interaction. As for PPI, there was no group main effect. However, the main SOA and attention effects were significant. The SOA $\times$ Attention interaction indicated that although PPF for both SOAs was higher for attend than for ignore trials, PPF in the attend condition was even higher during SOA 4,500 than during SOA 2,000.

\section{Baseline Startle and Habituation}

The magnitudes of the baseline startle responses during ITIs (see Table 2) did not differ between groups (means in $\mu \mathrm{V} \pm S D=$ $7.5 \pm 5.4$ for patients and $9.9 \pm 9.0$ for controls). However, they declined during the four ITI measurements $(\mathrm{T} 1=10.9 \pm 8.9$, $\mathrm{T} 2=9.1 \pm 8.2, \mathrm{~T} 3=7.7 \pm 7.5, \mathrm{~T} 4=7.3 \pm 6.6)$.

\section{Additional Analyses for the Verification of PPI and PPF Results}

Because there were more smoking patients than smoking controls (16 patients, 7 controls), $\chi^{2}(1, N=98)=4.6, p<.05$, we applied smoker status as an additional between factor into the PPI and PPF ANOVAs. We also conducted the ANOVAs only for the sample of nonsmokers. However, the effects remained as reported above (PPI SOA effect, PPI Group $\times$ Attention effect, PPF SOA effect, PPF attention effect, PPF SOA $\times$ Attention effect; all $F \mathrm{~s}>$ $\left.6.1, p \mathrm{~s}<.05, \eta_{p}^{2}>.06\right)$. With smoker status as an additional factor, the only change in the pattern of results entailed the loss of the SOA effect in the PPI analysis, $F(1,94)=2.3, p=.131$, $\eta_{p}^{2}=.02$. It is important to note that the Group $\times$ Attention effect in the PPI analysis did not interact with smoker status, $F(1,94)=$ $.005, p=.946, \eta_{p}^{2}<.001$. 
Table 2

Overview of PPI, PPF, Startle Habituation, and Error Analyses and Effects

\begin{tabular}{|c|c|}
\hline Effects and analyses & Statistics \\
\hline \multicolumn{2}{|l|}{ PPI } \\
\hline SOA & $F(1,96)=7.3, p<.01, \eta_{\mathrm{p}}^{2}=.07$ \\
\hline Attention & $F(1,96)=1.0, p=.310, \eta_{\mathrm{p}}^{2}=.01$ \\
\hline Group & $F(1,96)=0.6, p=.424, \eta_{\mathrm{p}}^{2}=.01$ \\
\hline Group $\times$ Attention & $F(1,96)=6.8, p<.05, \eta_{\mathrm{p}}^{2^{\mathrm{p}}}=.07$ \\
\hline Patients: Ignore vs. Attend & $t(48)=-1.0, p=.662$ \\
\hline Controls: Ignore vs. Attend & $t(48)=3.4, p<.001$ \\
\hline Ignore: Patients vs. Controls & $t(96)=-0.5, p=.621$ \\
\hline Attend: Patients vs. Controls & $t(96)=1.8, p=.246$ \\
\hline Group $\times$ SOA & $F(1,96)=0.3, p=.573, \eta_{\mathrm{p}}^{2}=.003$ \\
\hline SOA $\times$ Attention & $F(1,96)=0.01, p=.916, \eta_{\mathrm{p}}^{2}<.001$ \\
\hline Group $\times$ SOA $\times$ Attention & $F(1,96)=0.6, p=.436, \eta_{\mathrm{p}}^{2}=.01$ \\
\hline \multicolumn{2}{|l|}{ PPF } \\
\hline SOA & $F(1,96)=25.9, p<.001, \eta_{\mathrm{p}}^{2}=.21$ \\
\hline Attention & $F(1,96)=42.0, p<.001, \eta_{\mathrm{p}}^{2}=.31$ \\
\hline Group & $F(1,96)=0.2, p=.619, \eta_{\mathrm{p}}^{2^{\mathrm{p}}}=.003$ \\
\hline Group $\times$ Attention & $F(1,96)=2.3, p=.134, \eta_{\mathrm{n}}^{2}=.02$ \\
\hline Group $\times$ SOA & $F(1,96)=0.5, p=.467, \eta_{\mathrm{p}}^{2}=.01$ \\
\hline SOA $\times$ Attention & $F(1,96)=29.3, p<.001, \eta_{\mathrm{p}}^{2}=.23$ \\
\hline SOA 2,000: Ignore vs. Attend & $t(97)=-3.3, p<.01$ \\
\hline SOA 4,500: Ignore vs. Attend & $t(97)=-7.5, p<.001$ \\
\hline Ignore: SOA 2,000 vs. 4,500 & $t(97)=0.4, p=.725$ \\
\hline Attend: SOA 2,000 vs. 4,500 & $t(97)=-6.3, p<.001$ \\
\hline Group $\times$ SOA $\times$ Attention & $F(1,96)=0.5, p=.477, \eta_{\mathrm{p}}^{2}=.01$ \\
\hline \multicolumn{2}{|l|}{ ITI startle and habituation } \\
\hline Time of measurement & $F(3,288)=30.8, p<.001, \eta_{\mathrm{p}}^{2}=.24$ \\
\hline T1 vs. T2 & $t(97)=4.3, p<.001$ \\
\hline T1 vs. T3 & $t(97)=7.0, p<.001$ \\
\hline $\mathrm{T} 1$ vs. $\mathrm{T} 4$ & $t(97)=7.0, p<.001$ \\
\hline $\mathrm{T} 2$ vs. T3 & $t(97)=4.0, p<.001$ \\
\hline T2 vs. T4 & $t(97)=4.6, p<.001$ \\
\hline T3 vs. T4 & $t(97)=1.1, p=.261$ \\
\hline Group & $F(1,96)=2.7, p=.101, \eta_{\mathrm{p}}^{2}=.03$ \\
\hline Group $\times$ Time of Measurement & $F(3,288)=0.3, p=.789, \eta_{\mathrm{p}}^{2}=.003$ \\
\hline \multicolumn{2}{|l|}{ Overall error rate } \\
\hline Group & $F(1,96)=1.5, p=.218, \eta_{\mathrm{p}}^{2}=.02$ \\
\hline \multicolumn{2}{|l|}{ False alarms vs. misses } \\
\hline Group & $F(1,96)=1.5, p=.218, \eta_{\mathrm{p}}^{2}=.02$ \\
\hline Error & $F(1,96)=7.2, p<.01, \eta_{\mathrm{p}}^{2 \mathrm{p}}=.07$ \\
\hline Group $\times$ Error & $F(1,96)=0.004, p=.949, \eta_{\mathrm{p}}^{2}<.001$ \\
\hline \multicolumn{2}{|l|}{ Errors ignore vs. attend for PPI/PPF } \\
\hline Group & $F(1,96)=0.9, p=.334, \eta_{\mathrm{p}}^{2}=.01$ \\
\hline Attention & $F(1,96)=185.8, p<.001, \eta_{\mathrm{g}}^{2}=.66$ \\
\hline Prepulse condition & $F(1,96)=0.003, p=.953, \eta_{\mathrm{p}}^{2}<.001$ \\
\hline Group $\times$ Attention & $F(1,96)=0.6, p=.447, \eta_{\mathrm{p}}^{2}=.01$ \\
\hline Group $\times$ Prepulse Condition & $F(1,96)=0.03, p=.860, \eta_{\mathrm{p}}^{2}<.001$ \\
\hline Attention $\times$ Prepulse Condition & $F(1,96)=0.003, p=.954, \eta_{\mathrm{p}}^{2}<.001$ \\
\hline Group $\times$ Attention $\times$ Prepulse Condition & $F(1,96)=0.003, p=.954, \eta_{\mathrm{p}}^{2}<.001$ \\
\hline
\end{tabular}

Note. $\quad$ PPI $=$ prepulse inhibition; $\mathrm{PPF}=$ prepulse facilitation; $\mathrm{SOA}=$ stimulus onset asynchrony; $\mathrm{ITI}=$ intertrial interval; $\mathrm{T}=$ time of measurement.

Regarding comorbidity influences, we first conducted the PPI and PPF analyses with ADHD patients $(n=11)$ and controls $(n=$ 49 ) without comorbidity. In addition, we conducted the PPI and PPF analyses with any comorbidity (present/absent) as covariate. Finally, we subjected groups of psychiatric disorders (present/ absent) as covariates into the PPI and PPF analyses (affective disorders, anxiety disorders, addiction, smoking, eating disorders, Cluster A personality disorders, Cluster B personality disorders, and Cluster $\mathrm{C}$ personality disorders). For all analyses, the effects remained as reported (PPI SOA effect, PPI Group $\times$ Attention effect, PPF SOA effect, PPF attention effect, PPF SOA $\times$ Attention effect; all $\left.F \mathrm{~s}>4.7, p \mathrm{~s}<.032, \eta_{p}^{2}>.05\right)$.

\section{Error Rate}

The maximum achievable error rate was 48 (24 for ignore and 24 for attend trials). The ANOVA (see Table 2) revealed no group differences in overall error rate $\left(M_{\text {patients }}=5.3, S D=4.0 ; M_{\text {controls }}=\right.$ 4.4, $S D=3.2)$. Similarly, separate ANOVAs for false alarms $\left(M_{\text {patients }}=3.1, S D=3.1 ; M_{\text {controls }}=2.6, S D=2.3\right)$ and misses 
Table 3

Prepulse Startle Modulation (\%) According to Group, Attention Condition, and SOA

\begin{tabular}{|c|c|c|c|c|c|c|c|c|}
\hline \multirow[b]{3}{*}{ SOA } & \multicolumn{4}{|c|}{ Controls $(n=49)$} & \multicolumn{4}{|c|}{$\operatorname{ADHD}(n=49)$} \\
\hline & \multicolumn{2}{|c|}{ Ignore } & \multicolumn{2}{|c|}{ Attend } & \multicolumn{2}{|c|}{ Ignore } & \multicolumn{2}{|c|}{ Attend } \\
\hline & $M$ & $S D$ & $M$ & $S D$ & $M$ & $S D$ & $M$ & $S D$ \\
\hline 120 & -71.2 & 23.1 & -77.9 & 22.1 & -70.8 & 32.5 & -68.3 & 34.2 \\
\hline 240 & -63.1 & 28.1 & -72.8 & 24.7 & -67.6 & 24.4 & -62.9 & 40.9 \\
\hline 2,000 & 0.05 & 42.6 & 13.2 & 55.9 & -12.6 & 35.6 & 13.5 & 55.3 \\
\hline 4,500 & -1.3 & 33.5 & 43.4 & 70.1 & -13.8 & 32.4 & 53.3 & 77.0 \\
\hline
\end{tabular}

Note. $\quad \mathrm{SOA}=$ stimulus onset asynchrony $\mathrm{ADHD}=$ attention-deficit/hyperactivity disorder.

$\left(M_{\text {patients }}=2.2, S D=2.2 ; M_{\text {controls }}=1.8, S D=1.9\right)$ failed to find group differences. However, false alarms $(M=2.8, S D=2.7)$ were more frequent than misses $(M=2.0, S D=2.0)$, but this was marginal. There were no group differences for error rates when comparing errors in the ignore and attend trials for the PPI and PPF conditions, but errors in the attend condition $(M=3.9, S D=2.8)$ were more frequent than errors in the ignore condition $(M=0.1$, $S D=0.3$ ). Using error rates as covariates for the ANOVAs failed to affect the aforementioned pattern of effects for either PPI or PPF.

\section{Discussion}

This is the first study to systematically compare early and late processes of top-down controlled attention in adult ADHD using a prepulse startle paradigm. Our main finding was that ADHD patients showed a selective dysfunction of early controlled attentional processing, as indicated by the absence of a significant attention effect on PPI. This contrasted with intact controlled attentional processing at a later stage of information processing, as reflected in a normal attention effect on PPF. The main finding also contrasted with the lack of differences between patients and controls on PPI and PPF measured while participants were not required to attend to the prepulse.

Dysfunctional early controlled attention is in accordance with theories of ADHD as a disorder of top-down executive and effortful processes (Sergeant et al., 2003). Consistent with this are reports of ADHD-related derangements of the prefrontal cortex, the anterior cingulate cortex, and the thalamus. These structures are important for attentional control, including attentional modulation of PPI (Hazlett et al., 1998, 2001; MacDonald et al., 2000; Seidman et al., 2006; Tamm, Menon, \& Reiss, 2006). Dysfunctional early top-down controlled attentional processing was also reported for ADHD boys with respect to reduced event-related potentials to attended but not to ignored stimuli and with respect to the absence of an attentional effect on PPI (Hawk et al., 2003; Satterfield et al., 1994). Our results suggest that their findings may also apply to adults and to individuals from both gender groups. It therefore seems likely that the early attentional control dysfunction reflects a stable biopsychological indicator of ADHD.

It is also important that later top-down controlled attentional processes are intact in ADHD patients (PPF results). This suggests that deficient attentional control seen in the early phase of information processing (PPI results) disappears over time. Accordingly, controlled attentional processing may be slowed down in ADHD. A lag of several $100 \mathrm{~ms}$ behind normal attentional control could lead to a prolonged attentional blink or to errors in continuous performance tests ( $\mathrm{Li}$ et al., 2004; Song et al., 2005) and may be able to explain the slower performance of ADHD patients and their reduced temporal resolution (Bellgrove et al., 2006; Nigg et al., 2002). It needs to be examined whether the early attentional control dysfunction contributes to other cognitive impairments in memory and inhibitory dysfunctions as well.

It is important that no dysfunctions were noted in the nonattending test condition. Thus, automatic PPI and PPF appear to be intact in ADHD patients. This is in line with other studies using these paradigms in ADHD (Castellanos et al., 1996; Feifel et al., 2009; Hanlon et al., 2009; Hawk et al., 2003; Ornitz et al., 1992). Accordingly, ADHD does not seem to be a disorder of dysfunctional early and late automatic attentional processes or of controlled late attentional processes. This sets ADHD aside from other disorders, such as schizophrenia, with deficient automatic as well as controlled PPI and PPF (Hazlett et al., 2007; Wynn et al., 2004) and is consistent with the contrast in the severity of attentional dysfunctions between ADHD and schizophrenia.

Error rate was similar across groups. The behavioral task was not based on very short time intervals and therefore may be related to the absence of controlled attentional deficits in PPF. Similarly, we did not find differences in error rates between PPI and PPF trials. So deficient early top-down attentional control in ADHD patients was not simply due to a lack of compliance to the discrimination task on the PPI trials. Indeed, the low error rate in general points to an overall good compliance of the participants. Independent of group, we observed some more errors during attended compared with ignored trials, but this was expected because of some difficulty in distinguishing the 5-s and 7-s time intervals in the attend condition.

Our results are supported by strong effect sizes, strict individual matching procedures, additional analyses, and a pattern of reactivity in the controls that is comparable to that of previous studies. More specifically, the strong top-down controlled attentional modulation of PPI and PPF seen in our control participants was also noted previously (Filion et al., 1993; Heekeren, Meincke, Geyer, \& Gouzoulis Mayfrank, 2004). In general, PPI for SOA 120 was stronger than that during SOA 240. This may reflect a decrease of sensory gating over time. Indeed, it is functionally important that subsequent stimuli also are processed (Norris \& Blumenthal, 1996). The stronger PPF for SOA 4,500 compared with SOA 2,000 also fits with previous results (Hsieh, Swerdlow, \& Braff, 2006; Jennings et al., 1996). The interaction of SOA and attention 
indicated that the attention effect on PPF was stronger for SOA 4,500 compared with SOA 2,000, which shows that intentional orienting increases from 2,000 to $4,500 \mathrm{~ms}$ after the prepulse stimulus (Jennings et al., 1996).

Nevertheless, this is one of the first studies of its kind, so limitations and open questions need to be noted regarding our sample, procedures, and test parameters. Thus, our ADHD sample was marked by comorbidities. However, a high prevalence of comorbidities in ADHD is in accordance with previous findings (Kessler et al., 2006; Miller, Nigg, \& Faraone, 2007) and suggests that we investigated a representative sample. Furthermore, additional analyses showed that comorbidity did not account for the pattern of our results. Similarly, we did not obtain normative ratings of adult $\mathrm{ADHD}$ symptoms, nor was information from relatives always available. However, other data pointed to the clinical status of our patients, including a self-report of symptom severity, the fulfillment of $D S M-I V$ ADHD criteria, available collateral information, and a requirement that strong impairments be present in at least two settings. This also underscores the general complexity of diagnosis of $\mathrm{ADHD}$ and indicates that tests based on early attentional control may be used to improve the diagnosis of ADHD.

Regarding our test paradigm, we did not directly examine whether carryover effects might have influenced the relation between ignored and attended prepulses. ADHD patients have perseveration difficulties and seem to respond to stimuli which were previously associated with a task-specific significance, although they no longer have this meaning (Alderson, Rapport, Sarver, \& Kofler, 2008). However, patients and controls did not differ in prepulse startle modulation in ignored PPI and PPF trials for which carry-over effects should have resulted in increased PPI and PPF in ADHD patients. Participants were able to disengage their attention, because there was an absence of PPF in ignored trials (cf. Putnam \& Vanman, 1999). Furthermore, we observed a dysfunction in controlled attention in ADHD patients only for PPI, not for PPF. In addition, participants had $17.5 \mathrm{~s}$ between the presentation of the signal to attend/ignore and the prepulse to make themselves aware that the following acoustic stimuli can be ignored. Finally, the error data indicate that ADHD patients were well able to differentiate ignore and attend conditions.

Several issues emerge from this study. In healthy participants, controlled attentional prepulse startle modulation has been observed for a variety of short and long SOAs and seems to be influenced by the same selective attentional mechanisms. This points to a continuum of these controlled processes from early to late information processing (Elden \& Flaten, 2002; Jennings et al., 1996; Schell et al., 2000). We did not find attentional control dysfunctions in ADHD patients with longer time scales used in PPF. This isolates the deficit in top-down attentional modulation to rapid/early processes. However, we could not identify the time frame in which this deficit resolves. Future studies with SOAs between 240 and 2,000 ms may help to elucidate this question.

In addition, our test paradigm combines two different forms of modulation: PPI and PPF. The paradigm does confirm our model of attentional processing (see Figure 1), but it would be fruitful to apply the design to other neurobiological measures, such as functional imaging of brain areas involved in the relevant basic processes (e.g., prefrontal cortex). A further question is whether deficient early topdown controlled attentional processes in ADHD primarily reflect reduced motivation or effort. Thus, increases in arousal improve sustained attention performance in ADHD (O'Connell et al., 2008). Accordingly, future studies should focus on dissecting the nature of the demonstrated early control dysfunction. We suggest that therapeutic interventions intended to reduce attentional dysfunctions in ADHD would benefit from concentrating on these early controlled attentional dysfunctions. Thus, increasing arousal by neurofeedback or motivational factors may be useful strategies, because it has been shown that these ameliorate attentional dysfunctions in ADHD (O'Connell et al., 2008; Solanto, Wender, \& Bartell, 1997).

To summarize, our results strongly suggest that only subcomponents of attentional functions are disturbed in ADHD patients. Thus, ADHD is characterized by a dysfunction in early top-down controlled attentional processes, whereas automatic early and late attentional processing and controlled attentional processing at later stages seem to be normal.

\section{References}

Alderson, R. M., Rapport, M. D., Sarver, D. E., \& Kofler, M. J. (2008). ADHD and behavioral inhibition: A re-examination of the stop-signal task. Journal of Abnormal Child Psychology, 36, 989-998.

American Psychiatric Association. (1994). Diagnostic and statistical manual of mental disorders (4th ed.). Washington, DC: Author.

Bellgrove, M. A., Mattingley, J. B., Hawi, Z., Mullins, C., Kirley, A., Gill, M., \& Robertson, I. (2006). Impaired temporal resolution of visual attention and dopamine beta hydroxylase genotype in attention-deficit/ hyperactivity disorder. Biological Psychiatry, 60, 1039-1045.

Biederman, J., Mick, E., \& Faraone, S. V. (2000). Age-dependent decline of symptoms of attention deficit hyperactivity disorder: Impact of remission definition and symptom type. American Journal of Psychiatry, 157, 816-818.

Blumenthal, D. T. (1999). Short lead interval startle modification. In M. E. Dawson, A. M. Schell, \& A. Böhmelt (Eds.), Startle modification: Implications for neuroscience, cognitive science, and clinical science (pp. 51-71). Cambridge, England: Cambridge University Press.

Blumenthal, T. D., Cuthbert, B. N., Filion, D. L., Hackley, S., Lipp, O. V., \& van Boxtel, A. (2005). Committee report: Guidelines for human startle eyeblink electromyographic studies. Psychophysiology, 42, 1-15.

Braff, D. L., Geyer, M. A., \& Swerdlow, N. R. (2001). Human studies of prepulse inhibition of startle: Normal subjects, patient groups, and pharmacological studies. Psychopharmacology, 156, 234-258.

Castellanos, F. X., Fine, E. J., Kaysen, D., Marsh, W. L., Rapoport, J. L., \& Hallett, M. (1996). Sensorimotor gating in boys with Tourette's syndrome and ADHD: Preliminary results. Biological Psychiatry, 39, 33-41.

Douglas, V. I. (Ed.). (1999). Cognitive control processes in attention-deficit/ hyperactivity disorder. Dordrecht, The Netherlands: Kluwer Academic.

Elden, A., \& Flaten, M. A. (2002). The relationship of automatic and controlled processing to prepulse inhibition. Journal of Psychophysiology, 16, 46-55.

Ellwanger, J., Geyer, M. A., \& Braff, D. L. (2003). The relationship of age to prepulse inhibition and habituation of the acoustic startle response. Biological Psychology, 62, 175-195.

Feifel, D., Minassiana, A., \& Perrya, W. (2009). Prepulse inhibition of startle in adults with ADHD. Journal of Psychiatric Research, 43, 484-489.

Filion, D. L., Dawson, M. E., \& Schell, A. M. (1993). Modification of the acoustic startle-reflex eyeblink: A tool for investigating early and late attentional processes. Biological Psychology, 35, 185-200.

First, M. B., Spitzer, R. L., Gibbon, M., \& Williams, J. B. W. (1996). Structured Clinical Interview for DSM-IV Axis I Disorders, Clinician Version (SCID-CV). Washington, DC: American Psychiatric Press.

First, M. B., Spitzer, R. L., Gibbon, M., \& Williams, J. B. W. (1997). Structured Clinical Interview for DSM-IV Personality Disorders (SCID-II). Washington, DC: American Psychiatric Press.

Graham, F. K. (1975). The more or less startling effects of weak prestimulation. Psychophysiology, 12, 238-248.

Hanlon, M.-C., Karayanidis, F., \& Schall, U. (2009). Intact sensorimotor 
gating in adult attention deficit hyperactivity disorder. The International Journal of Neuropsychopharmacology, 12, 701-707.

Hawk, L. W., Yartz, A. R., Pelham, W. E., \& Lock, T. M. (2003). The effects of methylphenidate on prepulse inhibition during attended and ignored prestimuli among boys with attention-deficit hyperactivity disorder. Psychopharmacology, 165, 118-127.

Hazlett, E. A., Buchsbaum, M. S., Haznedar, M. M., Singer, M. B., Germans, M. K., Schnur, D. B., ... Troyer, B. T. (1998). Prefrontal cortex glucose metabolism and startle eyeblink modification abnormalities in unmedicated schizophrenia patients. Psychophysiology, 35, 186-198.

Hazlett, E. A., Buchsbaum, M. S., Tang, C. Y., Fleischman, M. B., Wei, T. C., Byne, W., \& Haznedar, M. M. (2001). Thalamic activation during an attention-to-prepulse startle modification paradigm: A functional MRI study. Biological Psychiatry, 50, 281-291.

Hazlett, E. A., Romero, M. J., Haznedar, M. M., New, A. S., Goldstein, K. E., Newmark, R. E., . . Buchsbaum, M. S. (2007). Deficient attentional modulation of startle eyeblink is associated with symptom severity in the schizophrenia spectrum. Schizophrenia Research, 93, 288-295.

Heekeren, K., Meincke, U., Geyer, M. A., \& Gouzoulis Mayfrank, E. (2004). Attentional modulation of prepulse inhibition: A new startle paradigm. Neuropsychobiology, 49, 88-93.

Hsieh, M. H., Swerdlow, N. R., \& Braff, D. L. (2006). Effects of background and prepulse characteristics on prepulse inhibition and facilitation: Implications for neuropsychiatric research. Biological Psychiatry, 59, 555-559.

Jacob, C., Romanos, J., Dempfle, A., Heine, M., Windemuth-Kieselbach, C., Kruse, A., . . Lesch, K. P. (2007). Co-morbidity of adult attentiondeficit/hyperactivity disorder with focus on personality traits and related disorders in a tertiary referral center. European Archives of Psychiatry and Clinical Neuroscience, 257, 309-317.

Jennings, P. D., Schell, A. M., Filion, D. L., \& Dawson, M. E. (1996). Tracking early and late stages of information processing: Contributions of startle eyeblink reflex modification. Psychophysiology, 33, 148-155.

Jonkman, L. M., Kenemans, J. L., Kemner, C., Verbaten, M. N., \& van Engeland, H. (2004). Dipole source localization of event-related brain activity indicative of an early visual selective attention deficit in ADHD children. Clinical Neurophysiology, 115, 1537-1549.

Kessler, R. C., Adler, L., Barkley, R., Biederman, J., Conners, C. K., Demler, O., ... Zaslavsky, A. M. (2006). The prevalence and correlates of adult ADHD in the United States: Results from the National Comorbidity Survey Replication. American Journal of Psychiatry, 163, 716-723.

Lehrl, S. (1989). Mehrfachwahl-Wortschatz-Intelligenztest MWT-B [Multiple choice word fluency test]. Erlangen, Germany: Perimed.

Li, C. S., Lin, W. H., Chang, H. L., \& Hung, Y. W. (2004). A psychophysical measure of attention deficit in children with attention-deficit/ hyperactivity disorder. Journal of Abnormal Psychology, 113, 228-236.

MacDonald, A. W., Cohen, J. D., Stenger, V. A., \& Carter, C. S. (2000, June 9). Dissociating the role of the dorsolateral prefrontal and anterior cingulate cortex in cognitive control. Science, 288, 1835-1838.

Miller, T. W., Nigg, J. T., \& Faraone, S. V. (2007). Axis I and II comorbidity in adults with ADHD. Journal of Abnormal Psychology, 116, 519-528.

Murphy, K., \& Barkley, R. A. (1996). Attention deficit hyperactivity disorder adults: Comorbidities and adaptive impairments. Comprehensive Psychiatry, 37, 393-401.

Nigg, J. T., Blaskey, L. G., Huang-Pollock, C. L., \& Rappley, M. D. (2002). Neuropsychological executive functions and DSM-IV ADHD subtypes. Journal of the American Academy of Child \& Adolescent Psychiatry, 41, 59-66.

Norris, C. M., \& Blumenthal, T. D. (1996). A relationship between inhibition of the acoustic startle response and the protection of prepulse processing. Psychobiology, 24, 160-168.

O'Connell, R. G., Bellgrove, M. A., Dockree, P. M., Lau, A., Fitzgerald, M., \& Robertson, I. H. (2008). Self-alert training: Volitional modulation of autonomic arousal improves sustained attention. Neuropsychologia, 46, 1379-1390

Ornitz, E. M., Hanna, G. L., \& de Traversay, J. (1992). Prestimulationinduced startle modulation in attention-deficit hyperactivity disorder and nocturnal enuresis. Psychophysiology, 29, 437-451.

Putnam, L., \& Vanman, E. J. (1999). Long lead interval startle modification. In M. E. Dawson, A. M. Schell, \& A. H. Boehmelt (Eds.), Startle modification: Implications for neuroscience, cognitive science, and clinical science (pp. 72-92). Cambridge, England: Cambridge University Press.

Retz-Junginger, P., Retz, W., Blocher, D., Stieglitz, R. D., Georg, T., Supprian, T., ... Rösler, M. (2002). Reliability and validity of the German short version of the Wender-Utah Rating Scale for the retrospective assessment of attention deficit/hyperactivity disorder. Nervenarzt, 74, 987-993.

Satterfield, J. H., Schell, A. M., \& Nicholas, T. (1994). Preferential neural processing of attended stimuli in attention-deficit hyperactivity disorder and normal boys. Psychophysiology, 31, 1-10.

Schell, A. M., Wynn, J. K., Dawson, M. E., Sinaii, N., \& Niebala, C. B. (2000). Automatic and controlled attentional processes in startle eyeblink modification: Effects of habituation of the prepulse. Psychophysiology, 37, 409-417.

Schulz, S. M., \& Alpers, G. W. (2007). EMGpeakfind: A MATLABtoolbox for scoring startle eye-blink, and other EMG data. In E. Wascher, M. Falkenstein, G. Rinkenauer, \& M. Grosjean (Eds.), Psychologie und Gehirn (p. 117). Available at http://hdl.handle.net/2003/24421

Seidman, L. J., Valera, E. M., Makris, N., Monuteaux, M. C., Boriel, D. L., Kelkar, K., . . Biederman, J. (2006). Dorsolateral prefrontal and anterior cingulate cortex volumetric abnormalities in adults with attentiondeficit/hyperactivity disorder identified by magnetic resonance imaging. Biological Psychiatry, 60, 1071-1080.

Sergeant, J. A., Geurts, H., Huijbregts, S., Scheres, A., \& Oosterlaan, J. (2003). The top and the bottom of ADHD: A neuropsychological perspective. Neuroscience \& Biobehavioral Reviews, 27, 583-592.

Silverstein, L. D., Graham, F. K., \& Calloway, J. M. (1980). Preconditioning and excitability of the human orbicularis oculi reflex as a function of state. Electroencephalography \& Clinical Neurophysiology, 48, 406-417.

Sobin, C., Kiley-Brabeck, K., \& Karayiorgou, M. (2005). Lower prepulse inhibition in children with the 22q11 deletion syndrome. American Journal of Psychiatry, 162, 1090-1099.

Solanto, M. V., Wender, E. H., \& Bartell, S. S. (1997). Effects of methylphenidate and behavioral contingencies on sustained attention in attentiondeficit hyperactivity disorder: A test of the reward dysfunction hypothesis. Journal of Child \& Adolescence Psychopharmacology, 7, 123-136.

Song, D. H., Shin, D. W., Jon, D. I., \& Ha, E. H. (2005). Effects of methylphenidate on quantitative EEG of boys with attention-deficit hyperactivity disorder in continuous performance test. Yonsei Medical Journal, 46, 34-41.

Swerdlow, N. R., Auerbach, P., Monroe, S. M., Hartston, H., Geyer, M. A., \& Braff, D. L. (1993). Men are more inhibited than women by weak prepulses. Biological Psychiatry, 34, 253-260.

Tamm, L., Menon, V., \& Reiss, A. L. (2006). Parietal attentional system aberrations during target detection in adolescents with attention deficit hyperactivity disorder: Event-related fMRI evidence. American Journal of Psychiatry, 163, 1033-1043.

Wender, P. H., Wolf, L. E., \& Wasserstein, J. (2001). Adults with ADHD. An overview. Annals of the New York Academy of Sciences, 931, 1-16.

Wynn, J. K., Dawson, M. E., Schell, A. M., McGee, M., Salveson, D., \& Green, M. F. (2004). Prepulse facilitation and prepulse inhibition in schizophrenia patients and their unaffected siblings. Biological Psychiatry, 55, 518-523.

Received February 20, 2009

Revision received March 5, 2010

Accepted March 8, 2010 